Sarah Sturrock, Emma Williams, Hemant Ambulkar, Theodore Dassios and Anne Greenough*

\title{
Maternal smoking and cannabis use during pregnancy and infant outcomes
}

https://doi.org/10.1515/jpm-2019-0422

Received November 14, 2019; accepted December 3, 2019; previously published online January 11, 2020

\section{Abstract}

Background: Our aim was to determine the prevalence of tobacco smoking and e-cigarettes and cannabis use during pregnancy, whether these were influenced by ethnicity, and their relationship to perinatal outcomes.

Methods: A study was carried out in 4465 infants whose mothers delivered during 2017 and 2018. Self-reported maternal smoking, e-cigarette and cannabis use at booking were recorded. Outcome measures were birthweight and head circumference z-scores and admission to the neonatal intensive care unit (NICU).

Results: Two hundred and five women reported smoking cigarettes $(4.7 \%)$, five were using e-cigarettes $(0.11 \%)$ and 106 were using cannabis (2.43\%). Women were most likely to smoke if young (15-19 years old) or from a mixed-race or White background. Cigarette smoking was associated with a lower mean z-score for birthweight $(-0.587$ vs. $-0.064)$ and head circumference ( -0.782 vs. -0.157$)$ (both outcomes $\mathrm{P}<0.0001)$. Young, mixed-race women were most likely to be both smoking and using cannabis during pregnancy and their infants had a lower birthweight mean

*Corresponding author: Prof. Anne Greenough, Neonatal Intensive Care Centre, $4^{\text {th }}$ Floor Golden Jubilee Wing, King's College Hospital NHS Foundation Trust, Denmark Hill, London SE5 9RS, UK; Department of Women and Children's Health, School of Life Course Sciences, Faculty of Life Sciences and Medicine, King's College London, London SE5 9RS, UK; The Asthma UK Centre for Allergic Mechanisms in Asthma, King's College London, London SE5 9RS, UK; and NIHR Biomedical Centre at Guy's and St Thomas NHS Foundation Trust and King's College London, London SE1 9RT, UK, Tel.: 0203299 3037, E-mail: anne.greenough@kcl.ac.uk. https://orcid.org/0000-0002-8672-5349

Sarah Sturrock, Emma Williams and Hemant Ambulkar: Department of Women and Children's Health, School of Life Course Sciences, Faculty of Life Sciences and Medicine, King's College London, London, UK

Theodore Dassios: Department of Women and Children's Health, School of Life Course Sciences, Faculty of Life Sciences and Medicine, King's College London, London, UK; and Neonatal Intensive Care Centre, King's College Hospital NHS Foundation Trust, London, UK $\mathrm{z}$ score $(-0.989$ vs. $-0.587, \mathrm{P}=0.028)$ and head circumference $\mathrm{z}$ score $(-1.33$ vs. $0.782, \mathrm{P}=0.025)$ than cigarette use alone.

Conclusion: Young, mixed-race women were most likely to be both smoking and using cannabis during pregnancy and should be targeted for cessation programmes.

Keywords: birthweight; cannabis; cigarette smoking; e-cigarettes; head circumference.

\section{Introduction}

Maternal smoking during pregnancy apparently remains common. Approximately one in $10(10.8 \%)$ mothers in the UK were reported to be smoking at the time of delivery in 2017/18; this had declined from $15.8 \%$ in 2006/07 [1]. Maternal smoking has been shown to have numerous deleterious effects including an increased rate of preterm birth and fetal growth restriction, increased rate of admission to the neonatal intensive care unit (NICU) and adverse effects on neurodevelopment. Maternal smoking cessation programmes have been associated with an absolute reduction in the number of smoking mothers of between three and six in 100 mothers, but have only been shown to increase birthweight with no reductions in NICU admissions or perinatal or neonatal mortality [2]. Although e-cigarettes are a possible aid to smoking cessation [3], there are concerns about the safety of their use during pregnancy due to the ability of nicotine to cross the placental barrier and cause oxidative stress and inflammation in the fetus [4].

Cannabis use has been associated with lower birthweight [5, 6], more admissions to the neonatal unit [5] and a higher prevalence of preterm births [6]. A meta-analysis, however, found no significant association between cannabis use and adverse neonatal outcomes following adjustment for tobacco smoking and socioeconomic deprivation [7]. The prevalence of maternal cannabis smoking in the UK has been reported to be $2.5 \%$ [8], but the impact on neonatal morbidity is unclear with only one study in the previous meta-analysis using data from the UK [7]. The results from that UK study suggested that maternal cannabis use was not associated with an increase in perinatal morbidity or mortality [9]. Our previous study examining 
positive pregnancy test urine samples in 1999, however, found that $14.5 \%$ were positive for cannabis and infants born to mothers with positive drug tests for cannabis had a significantly lower birthweight than those with negative tests [6].

The aim of this study was to determine the current prevalence of tobacco smoking and e-cigarettes and cannabis use, whether these were influenced by ethnicity, and their relationship to important perinatal outcomes, that is not only NICU admission and birthweight, but also head circumference.

\section{Materials and methods}

The outcomes of term, live-born infants (37-41 weeks of gestational age) delivered at an NHS Foundation Trust between $1^{\text {st }}$ August 2017 and $31^{\text {st }}$ July 2018 were analysed. Patients were excluded from the analysis if their neonatal records were unavailable. Patients were identified via Badgernet, an electronic maternity and neonatal patient records system. Anonymised data were extracted from maternity booking data and from the medical records of both mother and infant.

Ethical approval: The research related to human use complied with all the relevant national regulations, institutional policies and is in accordance with the tenets of the Helsinki Declaration. As this project was a clinical audit, ethical approval from a Research Ethics Committee was not required. The audit was registered with and approved by the author's institutional Audit Department.

Informed consent: This was a clinical audit, so informed written consent was not required.

Demographic information collected from maternal records included age, ethnicity, number of previous pregnancies and live births. Ethnicity, as recorded in maternal records, was categorised according to the Office of National Statistics groups: White, mixed/ multiple ethnic groups, Asian/Asian British, Black/African/Caribbean/Black British and other ethnic groups [10]. Cigarette smoking and e-cigarettes and cannabis use at the time of booking were recorded. Delivery records were reviewed to obtain the birthweight and head circumference, mode of delivery and APGAR scores at 1, 5 and 10 mins. Neonatal records were reviewed to determine whether the infant was admitted to the NICU, high-dependency unit, special care baby unit or transitional care.

\section{Statistical analysis}

Birthweight and head circumference z-scores were calculated on Microsoft Excel (Microsoft Corporation, Redmond, WA, USA) using the least mean square (LMS)-growth calculator add-in and the UK World Health Organisation data for birthweight and head circumference [11]. The difference in birthweight and head circumference z-scores between exposed and unexposed groups were analysed for statistical significance using Student's $t$-tests. Differences in exposure rates between age and ethnicity groups were analysed using Chi-squared $\left(\chi^{2}\right)$ tests. IBM SPSS Statistics for Macintosh, Version 25.0 (IBM, Armonk, NY, USA) was used.

\section{Results}

Four thousand three hundred and sixty-six women delivered live term babies during the study period. Four infants were excluded from the analysis as their neonatal notes were unavailable. The mean age of the mothers of the remaining 4362 infants was 32.5 years and the most commonly reported ethnicity was White $(n=2126)$. There was no record of smoking status in 46 women's records (1.05\%), and no record of drug use status in 178 women's records (4.28\%).

There was a significant difference in smoking rates according to ethnicity $(\mathrm{P}=0.001)$; women were most likely to smoke if from a mixed-race or White background compared to those from an Asian, Black or other ethnic backgrounds (Table 1). Mixed-race women were more likely to be using cannabis (5.43\%) when compared to Asian women $(0.92 \%)(\mathrm{P}=0.009)$ or Black women $(2.4 \%)$ $(\mathrm{P}=0.017)$. Younger women were significantly more likely to smoke cigarettes. The youngest age group (15-19 years, $13.46 \%$ smoking) was almost 7 times more likely to smoke than the oldest age group ( $40+$ years, $1.90 \%$ smoking, $\mathrm{P}<0.0001$ ).

Of the 206 women who reported smoking at booking, 166 were offered a referral to smoking cessation services, and 97 (58.43\%) accepted the referral.

Cigarette smoking was associated with a lower mean z-score for birthweight ( -0.587 vs. -0.064$)$ and head circumference $(-0.782$ vs. -0.157$)$ than using neither cigarettes nor cannabis (both outcomes $\mathrm{P}<0.0001$ ). Using cannabis alone was associated with a lower mean $\mathrm{z}$ score than neither cannabis nor cigarette use for birthweight $(-0.255$ vs. 0.064$)$ and head circumference $(-0.393$ vs. $-0.157)$, but neither of these differences were statistically significant $(\mathrm{P}=0.087$ and $\mathrm{P}=0.129$, respectively). Cannabis and cigarette use were associated with both a significantly lower birthweight $\mathrm{z}$ score $(-0.989$ vs. $-0.587, \mathrm{P}=0.028)$ and head circumference $\mathrm{z}$ score $(-1.33$ vs. $0.782 \mathrm{z}$ scores, $\mathrm{P}=0.025)$ than cigarette use alone. Young, mixed-race women were most likely to be both smoking and using cannabis during pregnancy, and their infants had a lower birthweight mean $\mathrm{z}$ score $(-0.989$ vs. $-0.587, \mathrm{P}=0.028)$ and head circumference $\mathrm{z}$ score $(-1.33$ vs. $0.782, \mathrm{P}=0.025)$ than cigarette use alone.

Significant differences were found in the birthweight z-scores between infants whose mothers did not smoke and those who smoked $<5$ cigarettes/day (mean difference $=0.587, \mathrm{P}<0.0001$ ), $6-10$ cigarettes $/$ day (mean difference $=0.590, P<0.0001$ ), $11-20$ cigarettes $/$ day (mean difference $=0.457, \quad \mathrm{P}=0.049$ ) or 21-30 cigarettes/day (mean difference $=1.13, \mathrm{P}=0.041$ ). Similarly, significant 
Table 1: Smoking and cannabis use related to ethnicity and maternal age.

\begin{tabular}{lrrr}
\hline & Total & $\begin{array}{r}\text { Number smoking } \\
\text { cigarettes (\%) }\end{array}$ & $\begin{array}{r}\text { Number using } \\
\text { cannabis (\%) }\end{array}$ \\
\hline Ethnicity & & & \\
White & 2126 & $121(5.7)$ & $46(2.20)$ \\
Mixed/multiple ethnic groups & 221 & $17(7.7)$ & $12(5.4)$ \\
Asian/Asian British & 218 & $3(1.4)$ & $2(0.9)$ \\
Black/African/Caribbean/Black British & 1250 & $46(3.7)$ & $30(2.4)$ \\
Other ethnic group & 233 & $6(2.6)$ & $6(2.6)$ \\
Not stated & 318 & & $8(15.4)$ \\
Maternal age, years & 4366 & & $21(6.1)$ \\
15-19 & 52 & $4(13.5)$ & $14(1.8)$ \\
$20-24$ & 344 & $43(13.7)$ & $42(2.7)$ \\
$25-29$ & 783 & $67(4.3)$ & $20(1.6)$ \\
$30-34$ & 1562 & $34(2.7)$ & $1(0.3)$ \\
$35-39$ & 1256 & $7(1.9)$ & \\
$40+$ & 369 & & \\
\hline
\end{tabular}

Data are represented as the mean (standard deviation).

differences were identified in head circumference z-score between infants whose mothers did not smoke and those who smoked $<5$ or $6-10$ cigarettes (mean differences $=0.693$ and 0.592 , respectively, $\mathrm{P}<0.0001$ ).

Four hundred and eighty-seven of the infants (11.31\%) required admission to enhanced neonatal care: 112 were admitted to the NICU, 33 to the high-dependency unit, 199 to the special care baby unit and 143 to transitional care. No significant associations were found between maternal smoking or cannabis use and admission.

\section{Discussion}

We have demonstrated that both smoking cigarettes and cannabis use were associated with a significant reduction in birthweight and head circumference z-scores. We used z-scores to adjust for sex and gestational age to assess those outcomes. Cannabis use alone was not associated with a significant reduction in birthweight or head circumference z-score, but the combination of cannabis and cigarette smoking resulted in a significant decrease in both z-scores compared to cigarette smoking alone. Importantly, we believe for the first time, we have demonstrated that young, mixed-race women were most likely to be both smoking and using cannabis during pregnancy and their infants had significantly lower birthweight and head circumference $\mathrm{z}$ scores than cigarette use alone.

Maternal smoking is known to be associated with reduced birthweight. Prenatal smoke exposure causes chronic hypoxic stress in the fetus [12], likely due to early morphological placental changes [13] which lead to reduced maternal intervillous space and reduced volume and surface area of fetal capillaries [14]. Smoking tobacco during pregnancy, particularly continuing to smoke after the pregnancy, is associated with a reduction in fetal head circumference growth of $-0.56 \mathrm{~mm} /$ week [15]. Our finding that head circumference was negatively associated with maternal smoking is consistent with previous studies [16], although our study was unique in using z-scores to adjust head circumference measurements for gestational age and sex. Our results are of concern as small head circumference at birth and poor head circumference growth is associated with neurodevelopmental impairment [17]. We found that there were significant differences between smoking no cigarettes and smoking $<5$ or 6-10 cigarettes/day, although no dose-response effect of smoking was demonstrated. This may be due to the low numbers of women admitting to smoking a large number of cigarettes. Previous studies, however, have identified a similar effect, with the largest decrease in birthweight being seen at low levels of prenatal smoking [18]. This indicates that smoking cessation would benefit women admitting to smoking low numbers of cigarettes prenatally and the focus should be on complete cessation rather than reducing the number of cigarettes.

The mechanism of action by which cannabis may affect fetal growth and development is less well defined. Tetrahydrocannabinol (THC) is able to cross the placenta, but fetal THC concentrations have been found to be lower than maternal concentrations. Cannabis smoking produces 5 times as much carbon monoxide as smoking cigarettes, so it is postulated that cannabis may affect fetal oxygenation, thereby altering fetal growth and 
development [19]. A meta-analysis of the effect of cannabis found that it is not an independent risk factor for low birthweight or pre-term delivery [7]. Previous studies, however, have noted that it is difficult to separate the effect of cannabis use from concomitant tobacco smoking, other recreational drug use or socioeconomic status, particularly at high levels of cannabis use [7]. Our study found that cannabis alone did not cause a significant reduction in birthweight or head circumference z-score, but cannabis and cigarette smoking did reduce both measures further to cigarette smoking alone.

We found that mothers who were young were more likely to be smoking cigarettes, which is consistent with other UK data [20]. In our study, young women were also more likely to be using cannabis, and mixed-race women were more likely to be using cannabis and/or cigarettes. Previous studies have highlighted that maternal smoking in the UK is associated with socioeconomic deprivation and unplanned pregnancy [20]. A study of an Aboriginal birth cohort found that maternal smoking and cannabis use during pregnancy was more common amongst women with a lower level of education, unemployment during pregnancy and those who had begun childbearing at a young age [21]. It would be important to target smoking cessation programmes at those particularly at-risk groups. It is important to note that there is no evidence that nicotine replacement therapy used for smoking cessation in pregnancy has negative or positive impacts on birth outcomes [22].

A weakness of the present study is that we relied on self-report measures of maternal cigarette, e-cigarette and cannabis use. It has been highlighted that self-report measures have been found to underestimate cannabis exposure by as much as half in pregnant women [23]. Our 1999 study in the same London area found the rates of tobacco and cannabis use (34.3\% and $14.5 \%$, respectively) were based on urinalysis [6]. In a later study in 2008, however, we demonstrated that $34 \%$ of Caucasian women admitted to smoking by self-report [24]. The lower rates of smoking by self-report identified in the present study may, therefore, reflect the national trend of reduction in maternal smoking in recent years; less than $11 \%$ of women smoked at the time of delivery in 2017/18, compared to $16 \%$ in 2006/07 [1].

We intended to examine the association between e-cigarette use and neonatal outcomes, but only six women within our study admitted to using e-cigarettes and four were concurrently smoking conventional cigarettes. E-cigarettes may be perceived as less harmful than tobacco smoking [25]. Nevertheless, due to the perceived social stigma, some women feel uncomfortable using e-cigarettes in public especially during pregnancy [26]. It has been noted that healthcare providers less frequently assessed electronic nicotine delivery systems during prenatal visits compared to tobacco cigarette use [27]. Use of electronic cigarettes to quit smoking, however, may be common in women of reproductive age [28]. Hence, more research is required to determine the risks and benefits of electronic cigarette use during pregnancy.

A strength of the study is the use of z-scores to adjust for the expected variation in these outcomes with gestational age and sex of the infant. We also only analysed term births, eliminating the possibility that the associations with reduced birthweight were confounded by prematurity, a known consequence of maternal smoking during pregnancy [29]. A further strength was the large sample size. The retrospective approach enabled us to quantify how many women were asked about smoking and substance misuse and how many are offered referrals without the knowledge of an ongoing study affecting staff behaviour.

In conclusion, uniquely, we found a significant reduction in head circumference $\mathrm{z}$-score as well as birthweight z-score in those exposed to tobacco smoke and cannabis. Young, mixed-race women were most likely to be smoking and using cannabis during pregnancy. That group should be a key target for maternal smoking cessation programmes.

Author contributions: AG designed the study, analysed the data and was involved in the preparation of the manuscript and approved the final version. SS collected and analysed the data and was involved in the preparation of the manuscript and approved the final version. EW collected the data and was involved in the preparation of the manuscript and approved the final version. HA collected the data and was involved in the preparation of the manuscript and approved the final version. TD analysed the data and was involved in the preparation of the manuscript and approved the final version. All the authors have accepted responsibility for the entire content of this submitted manuscript and approved submission.

Author disclosure: The views expressed are those of the authors and not necessarily those of the NHS, the NIHR or the Department of Health.

Research funding: This research was supported by the National Institute for Health Research (NIHR) Biomedical Research Centre at Guy's and St Thomas' NHS Foundation Trust and King's College London.

Employment or leadership: None declared.

Honorarium: None declared. 
Competing interests: The funding organization(s) played no role in the study design; in the collection, analysis, and interpretation of data; in the writing of the report; or in the decision to submit the report for publication.

Conflict of interest: None declared.

\section{References}

1. Statistics on women's smoking at the time of delivery - England: Quarter 1, April 2018 to June 2018. NHS Digit [Internet] Available from: https://files.digital.nhs.uk/A6/504662/statwome-smok-time-deli-eng-q1-18-19-rep.pdf.

2. Lumley J, Chamberlain C, Dowswell T, Oliver S, Oakley L, Watson L. Interventions for promoting smoking cessation during pregnancy. Cochrane Database Syst Rev 2009;3:CD001055.

3. Hartmann-Boyce J, McRobbie H, Bullen C, Begh R, Stead LF, Hajek P. Electronic cigarettes for smoking cessation. Cochrane Database Syst Rev 2016;3:CD010216.

4. Whittington JR, Simmons PM, Phillips AM, Gammill SK, Cen R, Magann EF, et al. The use of electronic cigarettes in pregnancy. Obstet Gynecol Surv 2018;73:544-9.

5. Gunn JK, Rosales CB, Center KE, Nunez A, Gibson SJ, Christ C, et al. Prenatal exposure to cannabis and maternal and child health outcomes: a systematic review and meta-analysis. $\mathrm{Br}$ Med J Open 2016;6:e009986.

6. Sherwood RA, Keating J, Kavvadia V, Greenough A, Peters TJ. Substance misuse in early pregnancy and relationship to fetal outcome. Eur J Pediatr 1999;158:488-92.

7. Conner SN, Bedell V, Lipsey K, Macones GA, Cahill AG, Tuuli MG. Maternal marijuana use and adverse neonatal outcomes: a systematic review and meta-analysis. Obstet Gynecol 2016;128:713-23.

8. Zammit S, Thomas K, Thompson A, Horwood J, Menezes P, Gunnell D, et al. Maternal tobacco, cannabis and alcohol use during pregnancy and risk of adolescent psychotic symptoms in offspring. Br J Psychiatry 2009;95:294-300.

9. Fergusson DM, Horwood LJ, Northstone K. Maternal use of cannabis and pregnancy outcome. Br J Obstecty Gynecol 2002;109:21-7.

10. Office for National Statistics. Ethnic group, national identity and religion. 2016. Available from: https://www.ons.gov.uk/methodology/classificationsandstandards/measuringequality/ethnicgr oupnationalidentityandreligion\#introduction-to-collecting-dataon-ethnic-group-religion-and-national-identity.

11. Wright CM, Williams AF, Elliman D, Bedford H, Birks E, Butler G, et al. Using the new UK-WHO growth charts. Br Med J 2010;340:c1140.

12. Bush PG, Mayhew TM, Abramovich DR, Aggett PJ, Burke MD, Page KR. Maternal cigarette smoking and oxygen diffusion across the placenta. Placenta 2000;21:824-33.

13. Jauniaux E, Burton GJ. The effect of smoking in pregnancy on early placental morphology. Obstet Gynecol 1992;79:645-8.
14. Hofhuis W, de Jongste JC, Merkus PJ. Adverse health effects of prenatal and postnatal tobacco smoke exposure on children. Arch Dis Child 2003;88:1086-90.

15. Jaddoe VW, Verburg BO, De Ridder MA, Hofman A, Mackenbach JP, Moll HA, et al. Maternal smoking and fetal growth characteristics in different periods of pregnancy: The Generation R Study. Am J Epidemiol 2007;165:1207-15.

16. Inoue S, Naruse H, Yorifuji T, Kato T, Murakoshi T, Doi H, et al. Impact of maternal and paternal smoking on birth outcomes. J Public Health 2017;39:557-66.

17. Raghuram K, Yang J, Church PT, Cieslak Z, Synnes A, Mukerji A, et al. Head growth trajectory and neurodevelopmental outcomes in preterm neonates. Pediatrics 2017;40:e20170216.

18. England LJ. Measures of maternal tobacco exposure and infant birth weight at term. Am J Epidemiol 2001;153:954-60.

19. Behnke M, Smith VC. Prenatal substance abuse: shortand long-term effects on the exposed fetus. Pediatrics 2013;131:e1009-24.

20. Flower A, Shawe J, Stephenson J, Doyle P. Pregnancy planning, smoking behaviour during pregnancy, and neonatal outcome: UK millennium cohort study. BMC Pregnancy Childbirth 2013;13:238.

21. Brown SJ, Mensah FK, Ah Kit J, Stuart-Butler D, Glover K, Leane $C$, et al. Use of cannabis during pregnancy and birth outcomes in an Aboriginal birth cohort: a cross-sectional, population-based study. Br Med J Open 2016;6:e010286.

22. Coleman T, Chamberlain C, Davey MA, Cooper SE, LeonardiBee J. Pharmacological interventions for promoting smoking cessation during pregnancy. Cochrane Database Syst Rev 2015;12:CD010078.

23. Markovic N, Ness RB, Cefilli D, Grisso JA, Stahmer S, Shaw LM. Substance use measures among women in early pregnancy. Am J Obstet Gynecol 2000;183:627-32.

24. Rao H, Donaldson N, Dobson P, Hannam S, Rafferty GF, Greenough A. Maternal smoking, ethnic origin, association with birth weight and NICU admission. Arch Med Sci 2008;4:310-14.

25. Baeza-Loya S, Viswanath H, Carter A, Molfese DL, Velasque KM, Baldwin PR, et al. Perceptions about e-cigarette safety may led to e-smoking during pregnancy. Bull Menninger Clin 2014;78:243-52.

26. Bowker K, Orton S, Cooper S, Naughton F, Whitemore R, Lewis $S$, et al. Views on and experiences of electronic cigarettes: a qualitative study of women who are pregnant or have recently given birth. BMC Pregnancy Childbirth 2018;18:233.

27. Bhandari NR, Day KC, Payakachat N, Franks AM, McCain KR, Ragland D. Use and risk perception of electronic nicotine delivery systems and tobacco in pregnancy. Women's Health Issues 2018;28:251-7.

28. Oncken C, Ricci KA, Kuo CL, Dornelas E, Kranzler HR, Sankey $\mathrm{HZ}$. Correlates of electronic cigarettes use before and during pregnancy. Nicotine Tob Res 2017;19:585-90.

29. Banderali G, Martelli A, Landi M, Moretti F, Betti F, Radaelli G, et al. Short and long term health effects of parental tobacco smoking during pregnancy and lactation: a descriptive review. J Transl Med 2015;13:1-7. 